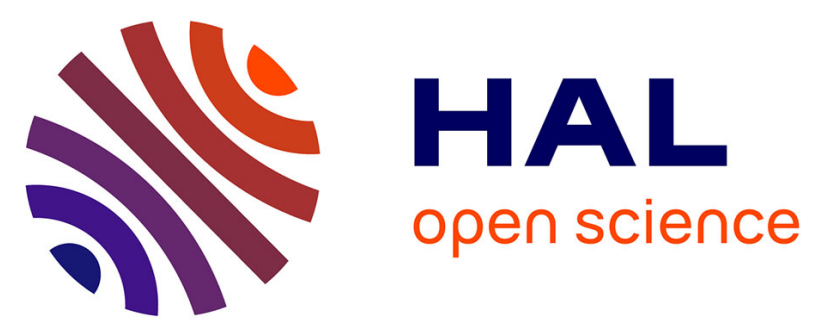

\title{
Les granitoïdes de la couverture protérozoïque de la bordure nord du craton du Congo (Sud-Est du Cameroun et Sud-Ouest de la République centrafricaine), témoins d'une activité magmatique post-kibarienne à pré-panafricaine
} Jean-Paul Vicat, Gaetan Moloto-A-Kenguemba, André Pouclet

\section{To cite this version:}

Jean-Paul Vicat, Gaetan Moloto-A-Kenguemba, André Pouclet. Les granitoïdes de la couverture protérozoïque de la bordure nord du craton du Congo (Sud-Est du Cameroun et Sud-Ouest de la République centrafricaine), témoins d'une activité magmatique post-kibarienne à pré-panafricaine. Comptes rendus de l'Académie des sciences. Série IIa, Sciences de la terre et des planètes, 2001, 332, pp.235-242. hal-00089828

\section{HAL Id: hal-00089828 \\ https://hal-insu.archives-ouvertes.fr/hal-00089828}

Submitted on 5 Mar 2013

HAL is a multi-disciplinary open access archive for the deposit and dissemination of scientific research documents, whether they are published or not. The documents may come from teaching and research institutions in France or abroad, or from public or private research centers.
L'archive ouverte pluridisciplinaire HAL, est destinée au dépôt et à la diffusion de documents scientifiques de niveau recherche, publiés ou non, émanant des établissements d'enseignement et de recherche français ou étrangers, des laboratoires publics ou privés. 


\title{
Les granitoïdes de la couverture protérozoïque de la bordure nord du craton du Congo (Sud-Est du Cameroun et Sud-Ouest de la République centrafricaine), témoins d'une activité magmatique post-kibarienne à pré-panafricaine
}

\author{
Jean-Paul Vicat ${ }^{\mathrm{a}, \mathrm{b}}$, Gaétan Moloto-A-Kenguemba ${ }^{\mathrm{c}}$, André Pouclet $^{\mathrm{b}, *}$ \\ a Département de géologie, université de N'Djaména, BP 1027, N'Djaména, Tchad \\ b Institut des sciences de la Terre, université d'Orléans, BP 6759, 45067 Orléans cedex 2, France \\ c Département de chimie-biologie-géologie, université de Bangui, BP 903, Bangui, République centrafricaine
}

Reçu le 7 septembre 2000 ; accepté le 15 janvier 2001

Présenté par Claude Jaupart

\begin{abstract}
Granitoids of the Proterozoic cover of the Congo craton northern edge (South-East of Cameroon and South-West of the Central African Republic), witnesses of a post-Kibarian to pre-Pan-African magmatic activity. Granitoid bodies dated from the Late Mesoproterozoic intrude the Palaeoproterozoic cover of the northern edge of the Congo craton. They line up a north-south left-lateral shear zone related to the Late Kibaran tectonics. They originated from crustal melting, may be due to the thermal anomalies, that were responsible of the large basaltic production during the pre-Pan-African extension of the Central Africa rift system. ๑ 2001 Académie des sciences / Éditions scientifiques et médicales Elsevier SAS
\end{abstract}

Cameroon / Republic of Central Africa / granitoids / Kibaran / Pan-African / Mesoproterozoic / Neoproterozoic

Résumé - Des pointements de granitoïdes datés de la fin du Mésoprotérozoïque intrudent la couverture paléoprotérozoïque du Nord du craton du Congo. Ils s'alignent sur un couloir de décrochement sénestre nord-sud, lié à la tectonique tardi-kibarienne. Ils proviennent d'une fusion crustale, probablement due aux anomalies thermiques qui ont généré d'importantes effusions basaltiques lors de l'extension pré-panafricaine du système de rifts de l'Afrique centrale. @ 2001 Académie des sciences / Éditions scientifiques et médicales Elsevier SAS

Cameroun / République centrafricaine / granitoïdes / Kibarien / Panafricain / Mésoprotérozoïque / Néoprotérozoïque

\section{Abridged version}

\section{Introduction - Geological setting}

At the Cameroon-Central African Republic (CAR) borders, numerous small granitoid bodies intrude the Palaeo- proterozoic cover of the Congo craton, namely the Sangha granites (Bolé, Libangué, Lopo, Yobé, and Kénié), the Kéka granodiorite, and the Monguélé syenite (figure 1). The Sangha granites line up a north-south left-lateral shear zone. The Yobé granite is dated at $1167 \pm 61 \mathrm{Ma}$ by $\mathrm{Rb} / \mathrm{Sr}$ method [2]. Its initial $\mathrm{Sr}$ isotopic ratio at 0.7342 sug-

\footnotetext{
* Correspondance et tirés à part.

Adresse e-mail : gestion.isto@univ-orleans.fr (A. Pouclet).
} 
gests a crustal origin. Many granite bodies are crosscut by dolerite dykes related to the pre-Pan-African extension around $1 \mathrm{Ga}[17,20]$. The host rocks, chloritoschists of the Bolé-Est series, are correlated with the Pama-Boda series in CAR, which is intruded by the Mbi granodiorite dated at $2070 \pm 70 \mathrm{Ma}$ by U/Pb method [10], and with the lower Dja series which overly the Archaean formations of the Congo craton. The Kéka granodiorite and the Monguélé syenite intrude the metapelites and sandstones of the Lobéké series and of the lower Dja series, also dated to Palaeoproterozoic. The syenite seems overlain by relics of the Tillitic complex dated to Cryogenian (850-650 Ma) $[1,16]$. Sm and $\mathrm{Nd}$ isotopic ratios give a $T_{\mathrm{DM}}$ at $1.54 \mathrm{Ga}$. The syenite being of mantellic origin (see below) emplaced between $1.54 \mathrm{Ga}$ and $850 \mathrm{Ma}$ and can be compared with the Sangha granites.

\section{Petrography and geochemistry}

New data concern the Lopo granite, the Kénié granite, and the Monguélé syenite. Granites are coarse-grained, with large zoned Na-plagioclase $\left(\mathrm{An}_{1.4-14.9}\right)$ and microcline phenocrysts, biotite $\left(\mathrm{Fe}^{\prime \prime} / \mathrm{Fe}^{\prime \prime}+\mathrm{Mg}=0.56-0.63\right.$; $\mathrm{TiO}_{2}=0.06-2.48 \%$ ), rare primary muscovite, titanite, apatite, magnetite, pyrrhotite and quartz. The Kénié granite is the most felsic. Granites suffered a mylonitic deformation as the effect of the nearby shear zone. Their compositions range from granite to monzogranites [11]. They are peraluminous [6] and calc-alkaline, as shown by the $\mathrm{Nb}-$, Ta- ant Ti-negative anomalies $\left(\mathrm{Nb} / \mathrm{La}_{\mathrm{N}}=0.10-0.28\right.$; $\left.\mathrm{Ti} / \mathrm{Tb}_{\mathrm{N}}=0.34-0.55\right)$ in the incompatible element normalized diagrams (figure 2). The syenite has an inequigranular texture with large $\mathrm{K}$-feldspars $\left(\mathrm{Or}_{72-97}\right)$, small albites $\left(\mathrm{An}_{0.1-2.8}\right), \mathrm{Mg}$ - and Ti-rich biotites $\left(\mathrm{Fe}^{\prime \prime} / \mathrm{Fe}^{\prime \prime} / \mathrm{Mg}=0.42-\right.$ $\left.0.43 ; \mathrm{TiO}_{2}=5.27 \%\right)$ and rare quartz. Secondary amphiboles (ferro-actinolites, ferrohornblendes, ferro-richterites, and ferro-eckermannites) developed at the expense of a previous magmatic sodic pyroxene. Composition is peral- kaline [6] as shown by the alkaline feature of the incompatible element normalized pattern (figure 2).

\section{Discussion and conclusion}

The granite geochemical signatures correspond to volcanic arc granites and to post-collision granites (figures 3 and 4). Meanwhile, the syenite is related to an intraplate anorogenic context. The orogenic signature of granites is inconsistent with the absence of Kibaran orogenic formations in the Sangha area. However, in the Late Mesoproterozoic to Early Neoproterozoic, important extension in Central Africa caused the opening of tectonic troughs of a great rift system [19]. The Sangha granites emplaced during strike slip motion of north-south fault bordering the Sangha graben, before the deposition of the syn-rift sediments (850-650 Ma) [1]. During the rift extension, important mafic magmatic activity of lithospheric and partly asthenospheric origin took place in so far as doleritic dyke swarms dated around $1 \mathrm{Ga}[17,19,20]$. Thus, large thermal anomalies have occurred. It is suggested that sialic melts generated by heating of the crust. Heat drained along a shear zone, from these anomalies or from mafic magma underplating. Few partial melt of the continental mantle also gave the syenite body.

Some aspects of this model concern the genesis of the Late Kibaran (1275-1249 Ma) A-type granites of Burundi [13]: asthenosphere heating along a shear zone. But, in the Sangha area, felsic melts are rather less abundant and are mainly crustal. On the contrary, Kibaran peraluminous crustal granites and post-Kibaran tin-bearing granites (around $1 \mathrm{Ga}$ ) are clearly different in showing syn-collision geotectonic signatures [3,13]. Similar crustal granites, dated around $1 \mathrm{Ga}$, are known in the WestCongolian area (figure 1, inset). They emplaced along a fault bordering the Mayombe rift where mafic magmatism took place. The same hypothesis is proposed [18]: crustal melt by heating along a fracture zone from asthenospheric uprising or from magma underplating.

\section{Introduction}

Aux confins du Cameroun et de la République centrafricaine (RCA), plusieurs petits pointements de granitoïdes traversent les formations attribuées au Paléoprotérozoïque qui recouvrent la bordure nord du craton archéen du Congo (figure 1).

Les granites de la Sangha sont localisés au sudouest de la RCA entre $2^{\circ} 75^{\prime}$ et $4^{\circ} \mathrm{N}$ et $16^{\circ}$ et $17^{\circ} \mathrm{E}$ (figure 1, site 4). On dénombre cinq massifs, du nord au sud : Bolé, Libangué, Lopo, Yobé et Kénié. Ces granites s'alignent sur un couloir de cisaillement sénestre nord-sud et intrudent les chloritoschistes de la série paléoprotérozoïque de la Bolé-Est [22]. Ils sont partiellement masqués par les dépôts phanérozoïques du bassin de Carnot. Les premières observations et données pétrographiques sont dues à Vincent [21], Wolf [22] et Censier [2]. Nous avons fait un nouvel échantillonnage des granites de Lopo et de Kénié.

À l'extrême Sud-Est du Cameroun, au moins deux massifs traversent, en plusieurs petits pointements, les chloritoschistes et les métagrésopélites des séries paléoprotérozoïques de la Lobeke et du Dja inférieur [4]. Ce sont la granodiorite de Kéka $\left(2^{\circ} 10^{\prime} \mathrm{N} ; 15^{\circ} 50^{\prime} \mathrm{E}\right)$ et la syénite de la Monguélé $\left(2^{\circ} 40^{\prime} \mathrm{N} ; 15^{\circ} 40^{\prime} \mathrm{E}\right)(f i$ gure 1 , sites 2 et 3 ). Nous nous sommes intéressés à la syénite. Par ailleurs, au sud de Monguélé, l'observation des images radar montre deux structures circulaires pouvant correspondre à des intrusions masquées par la couverture forestière.

Les questions posées concernent la composition pétrographique et chimique de ces granitoïdes et leur 


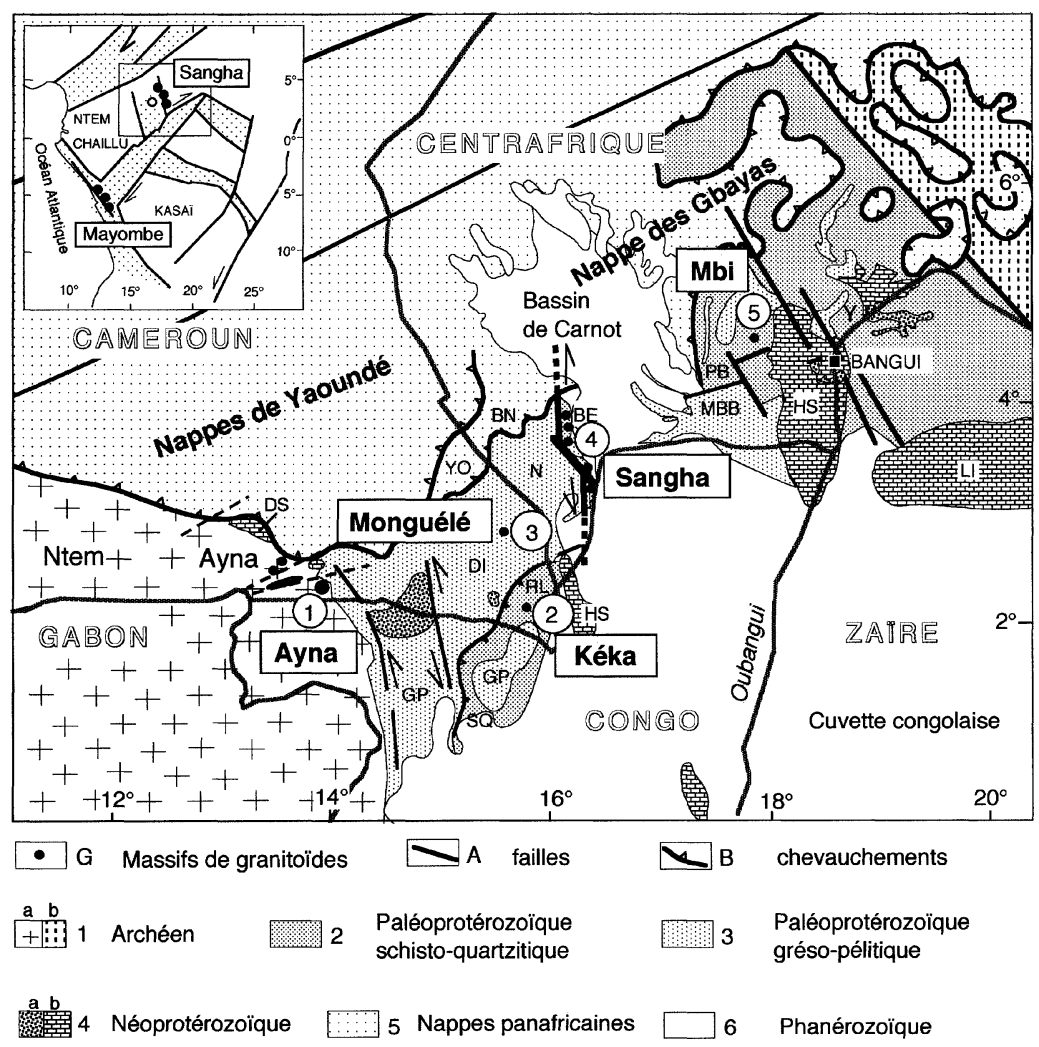

Figure 1. Schéma géologique des confins du Cameroun, Congo et RCA. G, massifs de granitoïdes et de syénite (1, granodiorites de l'Ayna; $\mathbf{2}$, granodiorite de Kéka ; $\mathbf{3}$, syénite de la Monguélé $; \mathbf{4}$, alignement des granites de la Sangha ; $\mathbf{5}$, granodiorite de Mbi). A, principaux décrochements. $\mathbf{B}$, chevauchements majeurs du Panafricain. 1, Archéen : a, socle de Ntem; b, socle de la RCA remobilisé au Panafricain. 2, séricitoschistes, micaschistes et quartzites paléoprotérozoïques (SQ, série schisto-quartzitique d'Ouesso ; RL, série de la ride de Lobéké; BE, série de la Bolé-Est ; PB, séries de Pama-Boda ; Y, série de la Yangana). 3, métagrès et métapélites paléoprotérozoïques (GP, série gréso-pélitique de Sembé ; DI, série du Dja inférieur; N, série de Nola; MBB, séries de M'Baïki-Bangui-Boali). 4, couverture néoprotérozoïque : a, tillites et grès fluvio-lacustres cryogéniens ; b, carbonates du Néoprotérozoïque III (DS, série du Dja supérieur ; HS, série carbonatée de la Haute Sangha ; LI, Lindien). 5, nappes panafricaines (BN, série de la Bolé-Nord; YO, série de Yokadouma). 6, dépôts phanérozoïques de la cuvette du Congo et du bassin de Carnot.

Encart. Aires décorées : fossés tectoniques d'âge Néoprotérozoïque au sein du craton du Congo d'après Vicat et al. [16], Alvarez [1] et Vicat et Pouclet [18]. Ronds pleins, granites de la Sangha et de la zone ouest-congolienne. Rond vide, syénite de la Monguélé.

Figure 1. Geological sketch of Cameroon, Congo and CAR borders. G, granitoid and syenite bodies (1, Ayna granites; 2, Kéka granodiorite; 3, Monguélé syenite; 4, Sangha granites; 5, Mbi granodiorite). A, main strike slip faults. B, main Pan-African thrusts. 1, Archaean: a, Ntem basement; b, CAR basement reworked by the Pan-African event. 2, Palaeoproterozoic sericitoschists, micaschists and quartzites (SQ, schistoquartzitic series of Ouesso; RL, ride de la Lobéké series; BE, Bolé-Est series; PB, Pama-Boda series: Y, Yangana series). 3, Palaeoproterozoic metasandstones and metapelites (GP, Sandstone-pelite series of Sembé; DI, lower Dja series; N, Nola series; MBB, M'Baïki-Bangui-Boali series). 4, Neoproterozoic cover: a, Cryogenian tillites and fluvio-lacustrine sandstones; b, Neoproterozoic III limestones (DS, upper Dja series; HS, Haute Sangha carbonated series; LI, Lindian). 5, Pan-African nappes (BN, Bolé-Nord series; YO, Yokadouma series). 6, Phanerozoic deposits of the Cuvette du Congo and of the Carnot basin.

Inset. Decorated area: Neoproterozoic tectonic grabens in the Congo craton, according to Vicat et al. [16], Alvarez [1] and Vicat and Pouclet [18]. Full circles, Sangha and West-Congolian granites. Open circle: Monguélé syenite.

signification géotectonique, compte tenu de leur âge Mésoprotérozoïque.

\section{Cadre géochronologique}

Le granite de Yobé est daté par $\mathrm{Rb} / \mathrm{Sr}$ à 1167 $\pm 61 \mathrm{Ma}$ [2]. Le rapport isotopique initial du $\mathrm{Sr}$ à 0,7342 traduit une origine crustale. Plusieurs des massifs granitiques de la Sangha sont recoupés par des dykes de dolérites à composition de tholéiites continentales, attribuées à la phase d'extension prépanafricaine qui se produit vers $1 \mathrm{Ga}[17,20]$. Les chloritoschistes encaissants de la Bolé-Est constituent le prolongement, sous les grès de Carnot, des schistes de la Pama-Boda de RCA dont la structuration et le métamorphisme paléoprotérozoïques sont scellés par l'intrusion de la granodiorite de Mbi (figure 1, site 5), datée par U/Pb à $2070 \pm 70 \mathrm{Ma}$ [10]. Ces séries reposant en discordance sur les formations archéennes du craton du Congo sont donc datées du Paléoproté- 
rozoïque. Il en est de même des séries métagrésopélitiques et métavolcano-sédimentaires de la Lobeke, du Dja inférieur et de Nola qui se prolongent au Congo par les séries schistoquartzitiques et grésopélitiques de Sembé-Ouesso [15].

La syénite de la Monguélé est apparemment antérieure au complexe tillitique [16] daté du Cryogénien (850-650 Ma) [1], qui repose en discordance sur les formations du Dja inférieur et dont on trouve des lambeaux près de la syénite. Les données isotopiques $\mathrm{Sm}-\mathrm{Nd}\left({ }^{238} \mathrm{Sm} /{ }^{144} \mathrm{Nd}=0,06980\right.$ et $\left.{ }^{143} \mathrm{Nd} /{ }^{144} \mathrm{Nd}=0,511705\right)$ permettent de calculer un $T_{\mathrm{DM}}$ à $1,54 \mathrm{Ga}$. La syénite, d'origine mantellique probable (voir plus bas), se met donc en place au Néoprotérozoïque, entre 1,54 Ga et $850 \mathrm{Ma}$, et peut être mise en parallèle avec les granites de la Sangha.

\section{Pétrographie}

Le granite de Lopo a une texture grenue porphyroïdique à gros grains. Il renferme des grands porphyroblastes de plagioclase sodique, de microcline perthitique et des amas de biotite chloritisée $\left(\mathrm{Fe}^{\prime \prime} / \mathrm{Fe}^{\prime \prime}+\right.$ $\left.\mathrm{Mg}=0,61-0,63 ; \mathrm{TiO}_{2}=1,45-1,72\right)$. Le reste de la paragenèse primaire comprend : quartz, titanite, apatite, ainsi que de la magnétite et de la pyrrhotite en grands cristaux. La muscovite secondaire et les épidotes $(\mathrm{Cz}=63,6 \%)$ sont en agrégats interstitiels. Des bandes de quartz engrenés à extinction roulante traduisent une déformation protomylonitique plus ou moins marquée. À l'approche du couloir de décrochement, la texture devient franchement mylonitique et orientée nord-sud sub-verticale. Le granite de Kénié présente les mêmes textures et composition minéralogique. Il est cependant plus pauvre en biotite $\left(\mathrm{Fe}^{\prime \prime} / \mathrm{Fe}^{\prime \prime}+\mathrm{Mg}=0,56-0,62 ; \mathrm{TiO}_{2}=0,06-\right.$ $2,48 \%$ ) et plus riche en microcline et en plagioclase sodique zoné $\left(\mathrm{An}_{1,4-14,9}\right)$. Les amas biotitiques contiennent quelques paillettes de muscovite primaire d'après leurs teneurs en $\mathrm{Mg}(0,26-0,73)$, Ti $(0,25-$ $0,15)$ et $\mathrm{Na}(0,05-0,06)$ [7]. La même déformation protomylonitique affecte la plupart des échantillons. Ces granites ont des paragenèses classiquement calcoalcalines, ce que confirme la composition des biotites [8]. Leur déformation indique une mise en place contemporaine du jeu transcurrent du grand décrochement nord-sud.

Les autres massifs sont pétrographiquement similaires. Le massif de Yobé se distingue par la présence de grenats [2].

La syénite de la Monguélé a une texture grenue hétérogranulaire, avec de grands cristaux de feldspath potassique $\left(\mathrm{Or}_{72-97}\right)$ perthitiques et de petits cristaux de plagioclase $\left(\mathrm{An}_{0,1-2,8}\right)$ et de quartz. Entre les feldspaths, se disposent de grandes paillettes de biotite magnésienne $\left(\mathrm{Fe}^{\prime \prime} / \mathrm{Fe}^{\prime \prime}+\mathrm{Mg}=0,42-0,43\right)$ et titanifère $\left(\mathrm{TiO}_{2}=5,27 \%\right)$, mais faiblement alumineuse $(\mathrm{Al}=2-2,7)$, comme il convient à des biotites de magma alcalin [8]. De la magnétite se développe au dépens des biotites et se trouve elle-même auréolée de titanite. À cette paragenèse magmatique primaire, se superpose une paragenèse secondaire de rétromorphose, à amphibole verte-chlorite-épidote. Les amphiboles sont en amas de gerbes fibreuses. Il s'agit d'amphiboles calciques : ferro-actinotes et ferrohornblendes $(\mathrm{Mg} / \mathrm{Mg}+\mathrm{Fe}=0,04-0,26)$ et calcosodiques : ferro-richtérites et ferro-eckermannites [5]. Les formes et clivages grossiers des amas à amphiboles ainsi que leurs compositions montrent qu'elles proviennent de la transformation d'un pyroxène magmatique sodique.

\section{Géochimie}

Le granite de Lopo est modérément siliceux $(64,1-$ $67,4 \%)$, alcalin $\left(\mathrm{Na}_{2} \mathrm{O}+\mathrm{K}_{2} \mathrm{O} / \mathrm{CaO}=2,9-4,9 \%\right)$ et ferromagnésien $(\mathrm{FeO}+\mathrm{MgO}=6,7-7,4)$ (tableau). Sa composition est celle des granites passant à des monzogranites [11]. Le contenu global en TR est modéré (214-397 ppm). Les profils normalisés à la chondrite sont très fractionnées $\left(\mathrm{La} / \mathrm{Yb}_{\mathrm{N}}=59-134\right)$, avec une anomalie négative marquée en $\mathrm{Eu}\left(\mathrm{Eu} / \mathrm{Eu}^{*}=0,4-0,6\right)$. Le granite de Kénié est plus riche en silice $(70,13-72,51 \%)$ et en alcalins $\left(\mathrm{Na}_{2} \mathrm{O}+\mathrm{K}_{2} \mathrm{O} / \mathrm{CaO}=6,8-10,0\right)$ et il est moins ferromagnésien $(\mathrm{FeO}+\mathrm{MgO}=3,3-4,2 \%)$. Il s'agit d'un granite commun. Un échantillon (BAY3), avec des teneurs faibles en $\mathrm{MgO}+\mathrm{FeO}(1,75 \%)$ et très élevées en alcalins $\left(\mathrm{Na}_{2} \mathrm{O}+\mathrm{K}_{2} \mathrm{O} / \mathrm{CaO}=20,1\right)$, a une composition de granite à feldspaths alcalins [11]. Le contenu global en TR est modéré à faible (107$342 \mathrm{ppm})$. Les profils normalisés à la chondrite sont très fractionnés $\left(\mathrm{La} / \mathrm{Yb}_{\mathrm{N}}=66-103\right)$, avec une anomalie négative peu marquée en $\mathrm{Eu}\left(\mathrm{Eu} / \mathrm{Eu}^{*}=0,7-0,9\right)$; l'échantillon BAY3 se distingue par une anomalie positive $\left(\mathrm{Eu} / \mathrm{Eu}^{*}=1,4\right)$.

En terme de signature géochimique, les granites de Lopo et de Kénié sont peralumineux [6] et calcoalcalins, comme le montrent les anomalies en $\mathrm{Nb}$ $\mathrm{Ta}\left(\mathrm{Nb} / \mathrm{La}_{\mathrm{N}}=0,10-0,28\right)$ et en $\mathrm{Ti}\left(\mathrm{Ti} / \mathrm{Tb}_{\mathrm{N}}=0,34\right.$ $0,55)$ sur les profils normalisés au manteau primitif (figure 2). Les anomalies en phosphore et en strontium sont typiquement liées au fractionnement cristallin.

La syénite de la Monguélé est peralcaline (A/CNK $=0,97)[6]$, avec une composition de syénite à feldspaths alcalins [11]. Le contenu global en TR (386 ppm) est voisin de celui du granite de Lopo et le profil normalisé à la chondrite est fractionné $\left(\mathrm{La} / \mathrm{Yb}_{\mathrm{N}}=47\right)$ sans anomalie en $\mathrm{Eu}\left(\mathrm{Eu} / \mathrm{Eu}^{*}=\right.$ 0,97). Le profil normalisé au manteau primitif 
J.-P. Vicat et al. / C. R. Acad. Sci. Paris, Sciences de la Terre et des planètes / Earth and Planetary Sciences 332 (2001) 235-242

Tableau. Composition chimique des granites de la Sangha et de la syénite de la Monguélé. Analyses réalisées au CRPG de Nancy par ICP pour les éléments majeurs et ICP-MS pour les éléments traces. LP6 à LP11 : granite de Lopo ; BAY2 à BAY5 : granite de Kénié ; PN996 : syénite de la Monguélé.

Table. Chemical compositions of the Sangha granites and of the Monguélé syenite. Analyses performed at the CRPG laboratory of Nancy by ICP for major elements, and by ICP-MS for minor elements. LP6 to LP11: Lopo granite; BAY2 to BAY5: Kénié granite; PN996: Monguélé syenite.

\begin{tabular}{|c|c|c|c|c|c|c|c|c|c|}
\hline \multirow[b]{2}{*}{ Référence } & \multicolumn{4}{|c|}{ Granite de Lopo } & \multicolumn{4}{|c|}{ Granite de Kénié } & \multirow{2}{*}{$\begin{array}{c}\text { Syénite de la Monguélé } \\
\text { PN996 }\end{array}$} \\
\hline & LP6 & LP9 & LP10 & LP11 & BAY2 & BAY3 & BAY4 & BAY5 & \\
\hline$\overline{\mathrm{SiO}_{2}}$ & 67,36 & 66,49 & 65,57 & 64,08 & 72,51 & 69,65 & 70,13 & 71,35 & 61,74 \\
\hline $\mathrm{TiO}_{2}$ & 0,60 & 0,73 & 0,67 & 0,69 & 0,28 & 0,13 & 0,34 & 0,40 & 0,86 \\
\hline $\mathrm{Al}_{2} \mathrm{O}_{3}$ & 15,03 & 14,94 & 15,02 & 16,10 & 14,38 & 16,68 & 15,18 & 14,51 & 15,69 \\
\hline $\mathrm{Fe}_{2} \mathrm{O}_{3}$ & 5,24 & 5,73 & 6,04 & 5,95 & 2,57 & 1,38 & 2,96 & 3,18 & 5,48 \\
\hline $\mathrm{MnO}$ & 0,02 & 0,04 & 0,03 & 0,03 & 0,03 & 0,01 & 0,02 & 0,01 & 0,04 \\
\hline $\mathrm{MgO}$ & 1,43 & 2,09 & 1,61 & 1,43 & 0,72 & 0,37 & 0,86 & 0,98 & 1,68 \\
\hline $\mathrm{CaO}$ & 1,60 & 1,56 & 1,92 & 2,56 & 0,77 & 0,52 & 1,17 & 1,03 & 1,08 \\
\hline $\mathrm{Na}_{2} \mathrm{O}$ & 2,84 & 3,14 & 2,97 & 3,06 & 3,65 & 3,61 & 3,87 & 3,69 & 4,65 \\
\hline $\mathrm{K}_{2} \mathrm{O}$ & 4,95 & 3,72 & 4,62 & 4,32 & 4,05 & 6,82 & 4,05 & 3,39 & 6,95 \\
\hline $\mathrm{P}_{2} \mathrm{O}_{5}$ & 0,24 & 0,30 & 0,28 & 0,27 & 0,08 & 0,04 & 0,09 & 0,10 & 0,16 \\
\hline $\mathrm{PF}$ & 1,01 & 1,21 & 1,22 & 1,45 & 1,12 & 0,86 & 1,29 & 1,32 & 1,29 \\
\hline Total & 100,32 & 99,95 & 99,95 & 99,94 & 100,16 & 100,07 & 99,96 & 99,96 & 99,62 \\
\hline $\mathrm{Ba}$ & 1001 & 1124 & 1022 & 965 & 1114 & 2235 & 1323 & 1193 & 2539 \\
\hline $\mathrm{Ce}$ & 157 & 102 & 100 & 187 & 79,4 & 50,9 & 127 & 173 & 184,3 \\
\hline $\mathrm{Co}$ & 8,8 & 13,3 & 12,8 & 11,1 & 4,6 & 2,4 & 5,5 & 5,8 & 10,6 \\
\hline $\mathrm{Cr}$ & 29,8 & 56,8 & 36,3 & 32,3 & 9,9 & 4,6 & 11,0 & 11,5 & 2,4 \\
\hline Dy & 2,45 & 4,86 & 2,15 & 3,05 & 1,33 & 0,86 & 1,78 & 2,23 & 3,66 \\
\hline $\mathrm{Er}$ & 0,73 & 2,18 & 0,60 & 0,79 & 0,50 & 0,34 & 0,78 & 0,87 & 1,89 \\
\hline $\mathrm{Eu}$ & 1,39 & 1,47 & 1,41 & 1,54 & 0,81 & 0,86 & 0,89 & 1,10 & 2,27 \\
\hline $\mathrm{Ga}$ & 20,0 & 21,2 & 20,6 & 23,0 & 19,4 & 18,9 & 21,9 & 20,9 & 19,0 \\
\hline $\mathrm{Gd}$ & 6,76 & 5,54 & 5,96 & 8,79 & 2,17 & 1,47 & 3,07 & 3,84 & 5,87 \\
\hline $\mathrm{Hf}$ & 5,70 & 7,68 & 6,76 & 6,05 & 4,20 & 3,22 & 6,08 & 7,54 & 9,55 \\
\hline $\mathrm{La}$ & 77,6 & 48,9 & 42,9 & 86,3 & 45,6 & 28,3 & 76,2 & 103 & 96,3 \\
\hline $\mathrm{Lu}$ & 0,07 & 0,20 & 0,08 & 0,10 & 0,06 & 0,06 & 0,10 & 0,12 & 0,22 \\
\hline $\mathrm{Nb}$ & 12,0 & 12,8 & 12,6 & 13,2 & 5,3 & 3,2 & 8,1 & 8,2 & 94,9 \\
\hline $\mathrm{Nd}$ & 65,2 & 45,8 & 40,6 & 73,9 & 25,8 & 16,1 & 42,2 & 54,1 & 61,4 \\
\hline $\mathrm{Ni}$ & 20,5 & 32,6 & 23,2 & 19,1 & 5,4 & 4,6 & 11,7 & 10,3 & 3,2 \\
\hline $\operatorname{Pr}$ & 18,7 & 12,4 & 10,7 & 20,5 & 7,8 & 5,0 & 13,4 & 17,7 & 18,5 \\
\hline $\mathrm{Rb}$ & 164 & 102 & 187 & 166 & 114 & 191 & 141 & 136 & 63 \\
\hline $\mathrm{Sm}$ & 9,74 & 7,24 & 7,94 & 13,10 & 3,51 & 2,37 & 5,65 & 6,86 & 8,69 \\
\hline Sn & 1,0 & 0,9 & 1,2 & 1,4 & 1,1 & 1,1 & 2,0 & 1,8 & 2,0 \\
\hline $\mathrm{Sr}$ & 257 & 153 & 310 & 387 & 175 & 207 & 226 & 230 & 547 \\
\hline $\mathrm{Ta}$ & 0,41 & 0,51 & 0,36 & 0,38 & 0,21 & 0,16 & 0,32 & 0,44 & 4,11 \\
\hline $\mathrm{Tb}$ & 0,70 & 0,84 & 0,60 & 0,83 & 0,29 & 0,19 & 0,41 & 0,47 & 0,73 \\
\hline Th & 45,1 & 18,0 & 14,8 & 42,0 & 35,5 & 18,8 & 29,4 & 38,9 & 18,3 \\
\hline V & 66,9 & 79,9 & 74,5 & 87,3 & 18,9 & 10,4 & 25,9 & 29,8 & 51,6 \\
\hline W & 0,16 & 0,27 & 0,08 & 0,15 & 0,19 & 0,04 & 0,06 & 1,49 & 0,34 \\
\hline $\mathrm{Y}$ & 7,5 & 24,6 & 7,5 & 9,1 & 6,4 & 4,5 & 10,9 & 9,3 & 18,8 \\
\hline $\mathrm{Yb}$ & 0,42 & 1,42 & 0,53 & 0,56 & 0,44 & 0,31 & 0,64 & 0,72 & 1,48 \\
\hline $\mathrm{Zn}$ & 51,9 & 68,5 & 66,7 & 58,5 & 48,6 & 30,7 & 69,7 & 68,5 & 6,2 \\
\hline $\mathrm{Zr}$ & 242 & 303 & 302 & 270 & 164 & 135 & 249 & 306 & 419 \\
\hline
\end{tabular}

(figure 2) montre un enrichissement croissant en éléments incompatibles et notamment lithophiles, caractéristiques des magmas alcalins, avec cependant une faible anomalie négative en $\mathrm{Ti}\left(\mathrm{Ti} / \mathrm{Tb}_{\mathrm{N}}=0,59\right)$ peutêtre d'origine pétrologique (déstabilisation des biotites titanifères). Les anomalies en phosphore et en strontium peuvent s'expliquer par du fractionnement cristallin.

\section{Interprétation géotectonique et discussion}

Les granites ont des rapports $\mathrm{FeO} / \mathrm{FeO}+\mathrm{MgO}$ pour des teneurs en $\mathrm{SiO}_{2}$ correspondant à des granites orogéniques [6], en accord avec leurs profils normalisés au manteau primitif (figure 2). Dans les diagrammes $\mathrm{Nb}$ vs $\mathrm{Y}$ et $\mathrm{Rb}$ vs $\mathrm{Y}+\mathrm{Nb}$ de Pearce 
J.-P. Vicat et al. / C. R. Acad. Sci. Paris, Sciences de la Terre et des planètes / Earth and Planetary Sciences 332 (2001) 235-242

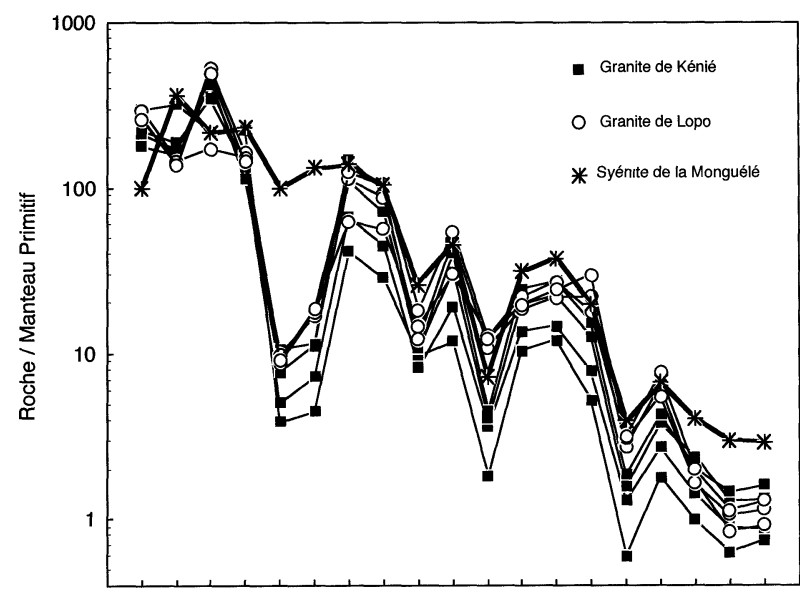

$\mathrm{Rb}$ BaTh K Ta Nb La Ce Sr Nd P Hf Zr Sm Ti Tb Y Yb Lu

Figure 2. Diagrammes de compositions normalisées au manteau primitif. Normalisation d'après Sun et McDonough [12].

Figure 2. Primordial Mantle-normalized diagrams. Values after Sun and McDonough [12].
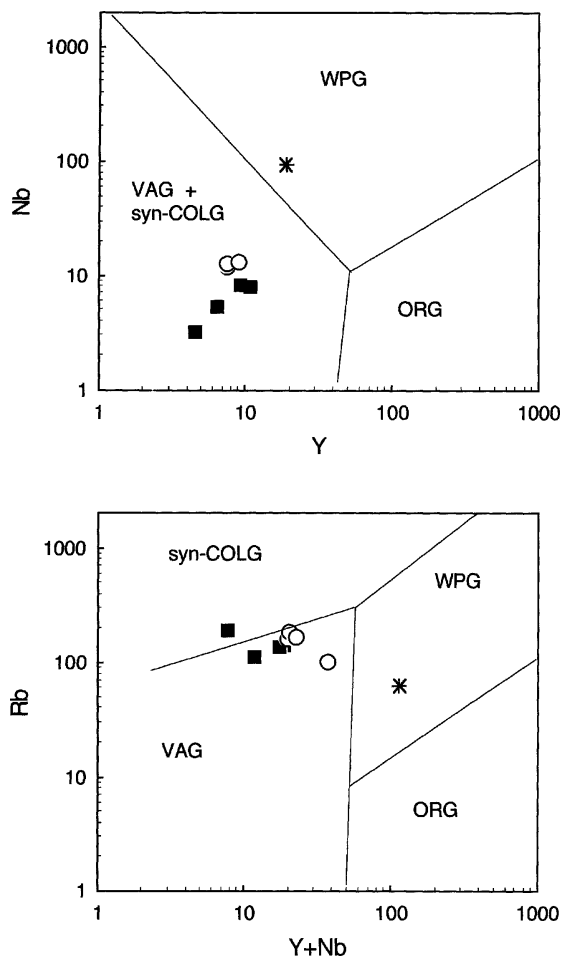

Figure 3. Diagrammes $\mathrm{Y}$ vs $\mathrm{Nb}$ et $\mathrm{Y}+\mathrm{Nb}$ vs $\mathrm{Rb}$ de Pearce et al. [9]. Mêmes symboles que sur la figure 2. WPG : granites intraplaques. ORG : granites des rides océaniques. VAG : granites d'arcs volcaniques. Syn-COLG : granites syn-collisionnels.

Figure 3. Diagrams $\mathrm{Y}$ vs $\mathrm{Nb}$ and $\mathrm{Y}+\mathrm{Nb}$ vs $\mathrm{Rb}$ of Pearce et al. [9]. Same symbols as for figure 2. WPG: within-plate granites. ORG: ocean ridge granites. VAG: volcanic arc granites. Syn-COLG: syncollision orogenic granites.

et al. [9], ils occupent le domaine des granites d'arc volcanique (VAG) (figure 3). Dans le diagramme $\mathrm{Zr}$ vs $(\mathrm{Nb} / \mathrm{Zr})_{\mathrm{N}}$ de Thieblemont et Tegyey [14] (figure 4), le granite de Lopo occupe le domaine des granites

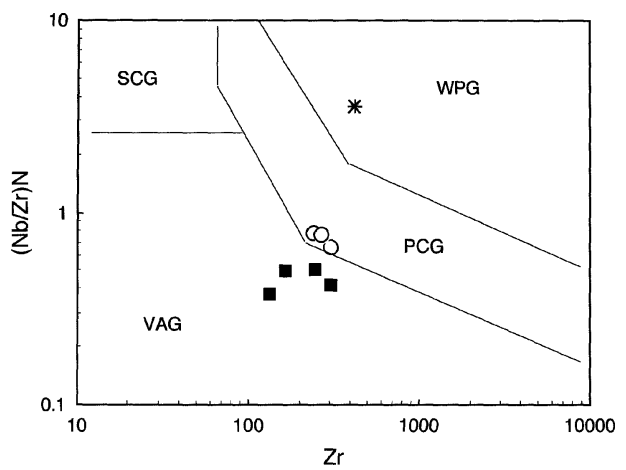

Figure 4. Diagramme $\mathrm{Zr}$ vs $(\mathrm{Nb} / \mathrm{Zr})_{\mathrm{N}}$ de Thiéblemont et Tégyey [14]. Mêmes symboles que sur la figure 2. WPG : granites des domaines intra-plaques. SCG : leucogranites alumineux syncollisionnels. PCG : granitoïdes post-collisionnels. VAG : granitoïdes associés aux zones de subduction.

Figure 4. Diagram $\mathrm{Zr}$ vs $(\mathrm{Nb} / \mathrm{Zr})_{\mathrm{N}}$ of Thiéblemont and Tégyey [14]. Same symbols as for figure 2. WPG: within-plate granites. SCG: syn-collision leucogranites. PCG: post-collision granitoids. VAG: volcanic arc granitoids.

orogéniques post-collisionnels, celui de Kénié, le domaine des VAG.

La syénite occupe, dans les diagrammes précédents, le domaine intraplaque caractéristique des magmas anorogéniques, en accord avec sa signature alcaline.

Le caractère orogénique des granites de la Sangha est en contradiction avec l'absence d'orogenèse kibarienne dans la région. Cependant, à la fin du Mésoprotérozoïque et au début du Néoprotérozoïque, on assiste à la formation des fossés tectoniques dans toute l'Afrique centrale et centro-occidentale [19]. Or, les granites de la Sangha se localisent sur l'une des failles majeures bordant l'aulacogène de la Sangha (figure 1, encart) qui appartient à ce réseau de fossés tectoniques et réutilise une fracturation méridienne héritée de la tectonique kibarienne. Mais ils sont antérieurs aux premiers dépôts néoprotérozoïques syn-rift (850-650 Ma) [1]. Cependant, la formation de ces fossés s'accompagne d'un important magmatisme en essaim de dykes doléritiques datés vers $1 \mathrm{Ga}[17,19$, 20]. Ces magmas basiques sont surtout des tholéiites continentales d'origine lithosphérique, avec une faible participation asthénosphérique. Cet important magmatisme mantellique traduit l'existence d'anomalies thermiques qui ont pu agir précocement sur le manteau et l'écorce par sous-placage des magmas basaltiques, bien avant les émissions en surface. Par drainage thermique le long des fractures majeures, notamment les grands décrochements sénestres nord-sud, comme celui de la Sangha, de la fusion crustale a pu se produire et générer les petits plutons granitiques de la Sangha remontés par diapirisme. Au niveau du manteau, une fusion plus modérée serait responsable de la genèse de la syénite de la Monguélé. 
Les granites de la Sangha se rapprochent des granitoïdes fini-kibariens de type A (i.e. d'affinité alcaline intra-plaque) du Burundi, datés à 1275 et à $1249 \mathrm{Ma}$, pour lesquels Tack et al. [13] invoquent une genèse par fusion mantellique le long d'un grand couloir transcurrent, à la suite d'un effondrement tardiorogénique, d'un épaississement crustal et de la remontée d'un coin asthénosphérique. Toutefois, pour la Sangha, seule de la fusion crustale se manifeste, avant les émissions fissurales basaltiques qui apparaîtront lors de l'extension franche des fossés. En revanche, les granites de la Sangha sont différents des granites peralumineux kibariens syn-orogéniques, comme des granites post-kibariens à étain, datés vers $1 \mathrm{Ga}$, mis en place dans un contexte transcurrent semblable [13], mais à nettes signatures syn-collisionnelles [3].

Des granites crustaux, assez similaires à ceux de la Sangha et datés vers $1 \mathrm{Ga}$, se localisent au CongoBrazzaville dans le domaine ouest-congolien, au niveau d'une faille bordière de l'aulacogène du Mayombe (figure 1, encart). Un magmatisme fissural basaltique se manifeste ensuite, avec l'extension du fossé [17], l'ensemble étant repris dans l'orogenèse ouest-congolienne. Là encore, il n'y a pas d'oroge- nèse kibarienne reconnue et la même hypothèse (fusion infra-crustale par drainage thermique le long de grandes fractures) est proposée [18].

\section{Conclusion}

Les granites de la Sangha de la fin du Mésoprotérozoïque seraient la manifestation précoce de l'apparition d'anomalies thermiques sous le craton d'Afrique centrale. Ce sont des liquides crustaux générés le long de couloirs transcurrents hérités de la tectonique kibarienne et ayant servi de drains thermiques. Dans le domaine orogénique kibarien du Burundi, ces anomalies ont provoqué d'importantes fusions mantelliques, à l'origine des granites de type $\mathrm{A}$, puis de la fusion crustale généralisée avec les granites peralumineux syn-orogéniques. En dehors de ce domaine, dans la Sangha comme dans la région ouest-congolienne, la fusion crustale est modérée et ne génère que quelques petits plutons mis en place entre 1,17 et $1 \mathrm{Ga}$. Les liquides mantelliques vont apparaître au début du Néoprotérozoïque, lors de la phase d'extension du système des rifts d'Afrique centrale, sous forme d'essaims de dykes doléritiques.

Remerciements. Nous remercions H. Martin pour la réalisation des analyses isotopiques de la syénite de Monguélé et P. Rolin pour sa lecture critique du premier texte.

\section{Références}

[1] Alvarez P., Les facteurs de contrôle de la sédimentation du supergroupe ouest-congolien (Sud-Congo). Rampe carbonatée et activité biologique au Protérozoïque supérieur, Mém. BRGM 239 (1995) 273.

[2] Censier C., Dynamique sédimentaire d'un système fluvuatile diamantifère mésozoïque. La formation de Carnot (République centrafricaine), thèse, université de Bangui, 1989, 591 p.

[3] Günther M.A, Dulski P., Lavreau J., Lehmann B., Möller P., Pohl W., The Kibaran tin granites: hydrothermal alteration versus plate tectonic setting, ICPG 255 Newsletter 2 (1989) 21-27.

[4] Laplaine L., Notice explicative sur la feuille Nola (partie Cameroun) de la carte de reconnaissance au 1:500000, Dir. Mines et géol. Cameroun, Yaoundé, 1971, 42 p.

[5] Leake B.E. et al., Nomenclature of amphiboles. Report of the Subcommittee on Amphibole of the International Mineralogical Association Commission on New Minerals and Mineral Names, Eur. J. Mineral. 9 (1997) 623-651.

[6] Maniar P.D., Piccoli P.M., Tectonic discrimination of granitoïds, Geol. Soc. Amer. Bull. 101 (1989) 635-643.

[7] Miller C.F., Sttodart E.F., Bradfish L.J., Dollase W., Composition of plutonic muscovite: genetic implications, Canadian Mineral. 19 (1981) 25-34.

[8] Nachit H., Razafimahefa N., Stussi J.-M., Carron J.-P., Composition chimique des biotites et typologie magmatique des granitoïdes, C. R. Acad. Sci. Paris, série II 301 (1985) 813-817.
[9] Pearce J.A., Harris N.B., Tindle A.G., Trace element discrimination diagrams for the tectonic interpretation of granitic rocks, J. Petrol. 25 (4) (1984) 956-983.

[10] Poidevin J.-L., Pin C., 2 Ga U-Pb zircon dating of Mbi granodiorite (Central African Republic) and its bearing on the chronology of the Proterozoic of Central Africa, J. Afr. Earth Sci. 5-6 (1986) 581-587.

[11] Streckeisen A., Le Maître R.W., A chemical approximation to he modal QAPF classification of igneous rocks, Neues Jb. Miner. Mont. 136 (1979) 169-206.

[12] Sun S.S., McDonough W.F., Chemical and isotopic systematics of oceanic basalts: implications for mantle composition and processes, in: Saunders A.D., Norry M.J. (Eds.), Magmatism in the ocean basins, Blackwell Sci. Publ., Oxford, 1989, pp. 313-345.

[13] Tack L, Liégeois J.-P., Deblond A., Duchesne J.-C., Kibaran A-type granitoids and mafic rocks generated by two mantle sources in a late orogenic setting (Burundi), Precambrian Res. 68 (1994) 322 356.

[14] Thiéblemont D., Tégyey M., Une discrimination géochimique de roches différenciées témoin de la diversité d'origine et de situation des magmas calco-alcalins, C. R. Acad. Sci. Paris, série II 319 (1994) 87-94.

[15] Vicat J.-P., Bilan des connaissances acquises sur les séries de Dja (Cameroun), Nola (Centrafrique) et Sembé-Ouesso (Congo), in: Vicat J.-P., Bilong P. (Eds.), Géosciences au Cameroun, Collect. GEOCAM, Press. Univ. Yaoundé I, 1/1998, pp. 369-383.

[16] Vicat J.-P., Moloto-A-Kenguemba G.R., La syénite de la Monguélé (SE Cameroun), in : Vicat J.-P., Bilong P. (Eds.), Géologie et environnements au Cameroun, Collect. GEOCAM, Press. Univ. Yaoundé I, 2/1999, pp. 387-396. 
[17] Vicat J.-P., Pouclet A., Nature du magmatisme lié à une extension pré-panafricaine : les dolérites des bassins de Comba et de Sembé-Ouesso, Bull. Soc. géol. France 166 (4) (1995) 355-364.

[18] Vicat J.-P., Pouclet A., Early and Late Proterozoic granitoids and rhyolites from the West-Congolian belt (Gabon, Congo, Cabinda, Bas-Zaïre, North Angola): chemical composition and geodynamical implications, J. Afr. Earth. Sci. (sous presse).

[19] Vicat J.-P., Gioan P., Albouy Y., Cornacchia M., Giorgi L., Blondin P., Mise en évidence, sur la bordure ouest du craton du Congo, de fossés d'effondrement d'âge Protérozoïque supérieur, masqués par les formations phanérozoïques de la cuvette du Zaïre, C. R. Acad. Sci. Paris, série II 309 (1989) 1207-1213.
[20] Vicat J.-P., Pouclet A., Nkoumbou C., Sémé Mouangué A., Le volcanisme fissural néoprotérozoïque des séries du Dja inférieur, de Yokadouma (Cameroun) et de Nola (Centrafrique) : signification géotectonique, C. R. Acad. Sci. Paris, série IIa 325 (1997) 671-677.

[21] Vincent P., Rapport sur la région de Nola, Arch. Dir. Mines Géol. Républ. Centrafricaine, 1952, 30 p. (non publié).

[22] Wolf J.P., Carte géologique de reconnaissance des Républiques centrafricaines, du Congo, Gabon et Tchad, à l'échelle du 1/500 000 (feuille Nola) avec notice explicative, Inst. Equat. Rech. Etudes Géol. Min., Brazzaville, 1962, 30 p. 